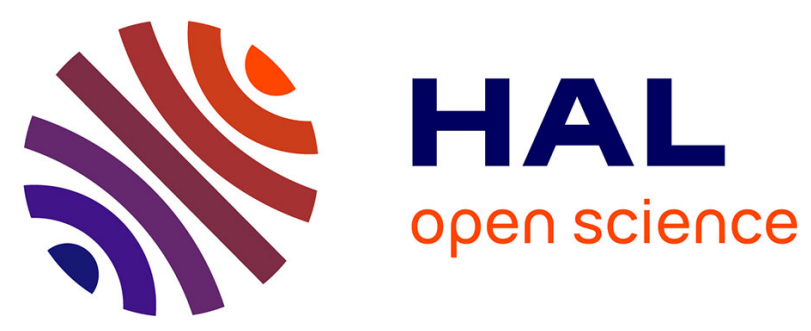

\title{
On-line state of charge estimation of embedded metal hydride hydrogen storage tank based on state classification
}

Dan Zhu, Youcef Ait Amirat, Abdoul Diaye, Abdesslem Djerdir

\section{- To cite this version:}

Dan Zhu, Youcef Ait Amirat, Abdoul Diaye, Abdesslem Djerdir. On-line state of charge estimation of embedded metal hydride hydrogen storage tank based on state classification. Journal of Energy Storage, 2021, 42, pp.102950 (9). hal-03359957

\section{HAL Id: hal-03359957 https://hal.science/hal-03359957}

Submitted on 30 Sep 2021

HAL is a multi-disciplinary open access archive for the deposit and dissemination of scientific research documents, whether they are published or not. The documents may come from teaching and research institutions in France or abroad, or from public or private research centers.
L'archive ouverte pluridisciplinaire HAL, est destinée au dépôt et à la diffusion de documents scientifiques de niveau recherche, publiés ou non, émanant des établissements d'enseignement et de recherche français ou étrangers, des laboratoires publics ou privés. 


\title{
On-line State of Charge Estimation of Embedded Metal Hydride Hydrogen Storage tank based on State Classification
}

\author{
Dan.Zhu ${ }^{\mathrm{a}, \mathrm{b}, *}$, Youcef.Ait-Amirat ${ }^{\mathrm{b}}$, Abdoul.N'Diaye ${ }^{\mathrm{b}}$, Abdesslem.Djerdir $^{\mathrm{b}}$ \\ ${ }^{a}$ School of Automobile, Chang'an University \\ $X i$ 'an 710064, Shaanxi Province, China \\ ${ }^{b}$ FEMTO-ST, CNRS, Univ. Bourgogne Franche-Comte, UTBM \\ FCLAB, CNRS, Univ. Bourgogne Franche-Comte \\ Rue Thierry Mieg, F-90010 Belfort Cedex, France
}

\begin{abstract}
With the further deterioration of environment and the depletion of fossil fuels, the alternative energy sources are urgently needed to be discovered. Hydrogen holds great promise thanks to its unlimited resources, high energy density and the environmentally friendly nature. However, its low volume density under normal temperature and pressure becomes the main challenge for on-board storage. Owing to its high potential of safety, one of the optimal solution for the future hydrogen vehicle is storing hydrogen using metal hydride (MH) under proper temperature and pressure. This work focuses on the state of charge (SOC) estimation of the embedded $\mathrm{MH}$ hydrogen storage tank. High precise estimating of the remaining energy will contribute to both the evaluation of reliability and the design of control strategy. A statistical model of SOC is proposed based on the database collected form laboratory experiments and real operation vehicle test. What's more, a joint multi-classifier is designed to recognize the current state of reaction. Under this condition, the SOC of MH hydrogen storage tank is calculated through combining the state classifier and SOC model. This proposed on-line SOC estimation procedure is validated with the real operation vehicles in both charging and discharging process. It is proved to be effective
\end{abstract}

\footnotetext{
${ }^{*}$ Corresponding author

Email address: dan.zhu@chd.edu.cn (Dan.Zhu)
}

Preprint submitted to Elsevier

July 10, 2021 
with the estimation error of $0.2 \%$ during charging and $4.29 \%$ of discharging.

Keywords: Metal hydride, Hydrogen storage, On-line SOC estimation, State classification

\section{Introduction}

Hydrogen, produced from renewable energy, is being evaluated and promoted worldwide as an ideal power source for its inexhaustibility, cleanliness, convenience and independence from foreign control, which make it as the replacement for gasoline, heating oil, natural gas, and other fuels in both transportation and non-transportation applications [1, 2, 3. For hydrogen vehicles, on-board hydrogen storage is one of the main challenges due to its low energy density per unit volume. Currently, hydrogen storage technologies including compressed hydrogen, liquefied hydrogen and hydrides, among them hydrogen storage in reversible metal hydrides $(\mathrm{MH})$ has received great attention as it offers the possibility to store hydrogen at low pressure and moderate temperature with high volumetric density 4 .

Hydride storage is material-based storage that hydrogen is physical or chemical absorbed reversibly by solid compounds under certain temperature and pressure conditions. During chemical sorption, chemical reaction occurs between hydrogen and material that hydrogen molecules are split into atoms and integrated with the storage material then generates hydride. The common used low temperature hydrides for hydrogen storage can be grouped based on the stoichiometries as $A B_{5}$-type (e.g. $\left.L a N i_{5}\right), A B_{2}$-type (e.g. $T i-Z r$ alloys), $A_{2} B$-type (e.g. $S b_{2} T i, S n_{2} C o$ ) and $A B$-type (e.g. $T i-F e$ alloys), where $A$ represents elements with high affinity for hydrogen typically rare-earth or alkaline earth metal (e.g., $C a, T i, Z r$, etc.) and $B$ represents elements with low affinity for hydrogen typically a transition metal that forms only unstable hydrides (e.g., $C r, M n, F e$, etc.) [5]. The quantities of hydrogen stored using metal hydride are quite large that the reaching volumetric density often higher than that of liquid hydrogen [6]. Besides, it holds potential benefits of security 

to host atomic ratio that:

$$
r=\frac{H}{X}=\frac{n_{H}}{n_{X}}=\frac{m_{H} / M_{H}}{m_{X} / M_{M H}}
$$

compared to compressed hydrogen storage tank [7]. Although the compressed and low temperature liquid hydrogen storage tanks have already been widely used on commercial vehicles while the application of metal hydrides hydrogen is limited by the cost and weight for the current stage, storing hydrogen with MHs is still a promising method for embed hydrogen storing on the future hydrogen vehicle thanks to its high potential of density, safe and reliable.

State of charge (SOC) estimation is always an important issue and great challenge for all the energy storage device like battery, super-capacitor, oil tank and gas tank. Monitoring the remaining energy like electric, oil or nature gas in the device precisely is quite important for the energy management strategy design and the vehicle power train reliability evaluation 8. Moreover, an accurate and efficient SOC estimation result reflects the health condition of the real applied energy storage device, which have a great impact on the control for practical operation in both charging and discharging process. Similarly, the SOC of a MH hydrogen storage tank should also be estimated to evaluate the remaining useful hydrogen. Especially for the design of an on-line SOC estimation method, from which the information of hydrogen mass storing in the tank is of great importance for the practical automobile application.

Generally, the hydrogen content of a MH sample is indicated by the hydrogen in which $r$ represents the host atomic ratio, $H$ and $X$ indicate hydrogen and the host material separately. In this equation, $n_{H}$ and $n_{X}$ are the molar number of the hydrogen absorbed in the $\mathrm{MH}$ sample and the host material respectively. $m_{H}$ and $m_{X}$ are the mass of the hydrogen absorbed in the $\mathrm{MH}$ sample and the host material respectively. $M_{H}$ is the molar mass of hydrogen and $M_{X}$ represents that of host material. As to an energy storage device, the SOC at each sampling time $\mathrm{i}$ is the percentage of the remaining energy $m(i)$ to the total 
Table 1 - List of symbols

\begin{tabular}{ll}
\hline Nomenclature & \\
\hline MH & Metal hydride \\
SOC & State of Charge $(\%)$ \\
SVM & Support Vector Machine \\
NB & Naive Bayes \\
FCHEV & Fuel cell hybrid electrical vehicle \\
P-C-T & Pressure-Composition-Temperature \\
$M_{H}$ & Molecular weight of hydrogen $(\mathrm{g} / \mathrm{mol})$ \\
$M_{M H}$ & Molecular weight of one kind of $\mathrm{MH}(\mathrm{g} / \mathrm{mol})$ \\
\hline Subscripts & \\
\hline ini & Initial State of the MH tank \\
end & Final state of the MH tank \\
\hline
\end{tabular}

amount of $m_{\text {total }}$, which can be written like the follow equation:

$$
S O C(i)=\frac{m(i)}{m_{\text {total }}} * 100 \%
$$

53

From literature works, researchers proposed several methods for investigating the hydrogenation properties of a solid hydrogen storage sample. A number of techniques are available to measure the hydrogen sorption capacity of a solid reactor including the measurements of hydrogen pressure, component volume, hydrogen flow and sample mass in a sample chamber, connected to a hydrogen source, a hydrogen sink and a gas manifold [9]. One can also quote the Sievert technique using the variation of pressure in a constant and calibrated system volume to determine the hydrogen storage capacity [10. It's well known for the advantages of cost-effective, easy to set up, simple, physically robust and reasonably reliable. However, researchers found it critically dependent on the accurate volume calibration, especially for the high pressure measurement [1]. Gravimetric method is a reliable tool to measure the absorbed hydrogen mass 
at high pressure condition, which determines the hydrogen sorption isotherm at equilibrium state via mass measurement [12. Secondary ion mass spectrometry and neutron scattering are techniques based on the chemical composition and physical structure analysis of the hydrogen storage materials. Using these method, except for the accurate hydrogen capacity measurement, detailed information of hydrogenating reaction can be achieved and wider scale of material can be tested [13, 14, 15. Nevertheless, strict experimental conditions and expensive equipment make it confined to the sample analysis in laboratory. These techniques are effective for the characterization of hydrogen and SOC estimation of hydrogen storage materials while it is not suitable for practical application of a hydrogen reactor, especially for the on-board hydrogen storage tanks in transportation applications. For these applications of a MH hydrogen storage reactor, the required SOC estimation method should be efficient, reversible and movable.

Designing and simulating of the mathematical model is also an effective method to observe the MH state variation during absorption or desorption process [16. Meanwhile, the relationship of the different effective factors like transport properties, equilibrium situations and reaction kinetics can be determined by the coefficients and functions [17, 18. Researchers have proposed some $\mathrm{MH}$ tank models to describe the reaction process. The first two-dimensional numerical model is proposed by A. Jemni et al to emphasize the effect of the shape, pressure and cooling system [19. What's more, the three-dimensional model figured out the parameters could be optimized to get an optimal storage, including the pressure, permeability and thermal conductivity of the hydride 20, 21. However, there are too many complex elements like heat transfer, metal hydride density should be taken into consideration in these models.

In the current stage of study for the embedded $\mathrm{MH}$ tank, the remaining hydrogen mass is generally calculated by the integration of hydrogen flow rate refueled in and released out. A gas flow sensor is well applied to measure the gaseous hydrogen. However, during long-term operation, the measurement error of flow sensor is accumulated along with the increased charging and discharging 
cycle numbers. The expanding error of hydrogen flow leads to the decline of estimation accuracy, it might resulting in an erroneous control strategy and an irreversible damage on the properties of the $\mathrm{MH}$ hydrogen storage tank or other related devices like fuel cell 22,23 . Besides, the uncertain information of the initial hydrogen concentration makes the SOC calculation inaccuracy. Thus, an effective on-line SOC estimation method is necessary to measure and calibrate the remaining hydrogen mass.

This work provides a new, simple, direct and effective method to evaluate the hydrogen content stored in a $\mathrm{MH}$ hydrogen storage tank based on its characterization and performance. The on-line SOC estimation process is developed with physical analysis, statistical modeling and state classification, which is validated by the database recorded on the real operation vehicles and proved to be useful and efficient. In our study, a statistical model reflecting the relationship between equilibrium pressure, temperature and SOC is proposed for describing the performance of hydrogen storage and estimating the hydrogen capacity. The operation condition and performance of the embedded hydrogen storage reactor is more complicated with real operation requirements. So, the proposed statistical model might not satisfy to the precision requirement of the SOC estimation mission at each time. In this study, the dynamic performance of the hydrogen tank is analysed, from which a certain period is found to be available to apply the statistical model for SOC estimation. Then, a state classifier is designed to identify this state to realise the on-line SOC estimation of a MH hydrogen storage tank embedded on fuel cell vehicle. Finally, the proposed on-line SOC estimation process is validated by the database recorded on the real operation vehicles in both charging and discharging situation.

\section{P-C-T based statistical model for SOC estimation}

In order to detect the features of the applied $\mathrm{MH}$ hydrogen storage tank on the vehicle, a test bench in laboratory is built. On this test bench, a group of validation database is collected using the similar way of D. Chabane did [24]. 
The experiments are carried out for both absorption and desorption reaction. In absorption process, the initial conditions were set as the ambient temperature and empty tank. The hydrogen flow rate is as low as $0.6 \mathrm{~kg} / \mathrm{h}$ for the purpose of avoiding high kinetics and limiting temperature variations. The experiments are carried out for both absorption and desorption reaction. In absorption process, the initial conditions were set as the ambient temperature and empty tank. During hydrogenation reaction, the exothermic absorption process causes the temperature in the tank to raise, which also lead to the pressure increase. By defining the threshold of temperature, the hydrogen mass flow filling into the tank was controlled, which will be stopped when the temperature reached the threshold. The system returns naturally to the ambient temperature after energy convection and heat transfer. This process of charge will be repeated several times until the tank is fully charged or completely empty. The desorption process is in opposite direction. During the test, the remaining hydrogen mass and the SOC of the MH tank are carefully controlled and calculated by the hydrogen flow rate. The cumulative error is considered to be artificially avoided. Thus, using a new tank in a strictly controlled experiment, the estimated SOC is reliable to be considered as the calibrate reference.

From this database one can find that the data points of pressure at same temperature are parts of a corresponding P-C-T isotherm of the tested $\mathrm{MH}$ hydrogen storage tank. For each P-C-T isotherm of the tested MH tank, the complete process of both hydrogenation and hydrogen extraction can be described in three phases, including start to increase phase, slowly increase phase and speedy increase phase. Moreover, all these P-C-T curves have the same trend 25$]$.

Correspondingly, the variation of the hydrogen mass absorbed by the $\mathrm{MH}$ tank presents three stages, namely, slowly increase stage, speedy increase stage and the stage of tend to be constant. During the first stage, the hydrogen concentration is too low to active the hydrogenation reaction in a high speed. Although the pressure increases rapidly, the absorption of hydrogen is slowly. It comes to the second stage when the hydrogen to metal ratio reaches a certain 
value, in which the reaction reaches to equilibrium state and advances smoothly. Thus, the hydrogen is absorbed in a high speed. When the MH tank is fully charged, the input hydrogen flow leads to the raise of pressure since no more hydrogen can be stored in the $\mathrm{MH}$ crystal. This feature of hydrogen mass variation is similar to the probability distribution function. Under this situation, the relationship among mass of hydrogen absorbed, pressure and temperature is able to be identified through the least squares method and the hydrogen mass can be directly described by the following equation, which means the statistical model to describe the variation of the hydrogen mass as a function of pressure $P$ can be expressed as:

$$
\text { Mass }=k 1+\frac{k 2}{1+\exp (k 3 * P+k 4)}
$$

In this equation, Mass represents the hydrogen mass stored by the $\mathrm{MH}$ tank and $P$ is the tank pressure. $k 1, k 2, k 3$ and $k 4$ are the coefficients reflecting the influence of temperature and durability on hydrogenation reaction. $k 1$ corresponds to the initial condition of the $\mathrm{MH}$ tank, ideally at the beginning of charging process, the tank is empty and $k 1$ is zero. $k 2$ corresponds to the hydrogen storage capacity of the MH tank, namely, the hydrogen mass stored in a fully charged tank. The coefficients $k 3$ and $k 4$ are effected by temperature $T$ and correspond to the equilibrium pressure in the tank, which will determine the shape of P-C-T isotherms.

In practical application of a MH hydrogen storage tank, the performance is also influenced by the state of health and the operation temperature. After larger number of charging and discharging cycles, the $\mathrm{MH}$ tank suffers from degradation so that the hydrogen storage capacity declines. Besides, the $\mathrm{MH}$ tank can not be completely discharged after degradation. Thus, the coefficient $k 1=\operatorname{mass}_{i n i}(n)$, which varies along with cycle number $n$. The capacity not only depends on the number of cycles $n$ determined by the effect of ageing, but also corresponds to temperature. So, the coefficient $k 2$ can be expressed as $\operatorname{mass}(n, T)$. At the end of a charging process, the whole mass of hydrogen 
stored in the $\mathrm{MH}$ tank mass $s_{\text {end }}$ can be calculated by $k 1+k 2$. In one cycle, the behaviour is determined by temperature. Therefore, the coefficients $k 3=f_{1}(T)$ and $k 4=f_{2}(T)$ are the functions of temperature.

The SOC of MH tank at each moment $i$ is the percentage of the available hydrogen mass stored in the tank to the total amount, which can be expressed as following equation:

$$
\operatorname{SOC}(i)=\frac{\operatorname{Mass}_{(i)}-\text { mass }_{\text {ini }}}{\text { mass }_{\text {end }}} * 100 \%
$$

where Mass $(i)$ corresponds to the mass of hydrogen extracted from the $\mathrm{MH}$ tank at this sampling time, mass ${ }_{i n i}$ represents the hydrogen mass rest in the crystal which can not be released under normal operation condition and mass $s_{\text {end }}$ is the hydrogen mass in a full tank.

Based on Eq. 3, the SOC model of a MH tank can be also expressed as follows:

$$
S O C(i)=\frac{1}{1+\exp \left(f_{1}(T) * P+f_{2}(T)\right)} * 100 \%
$$

It shows that the SOC of a MH tank is possible to be reflected by the pressure and temperature, no matter of the ageing degree. Thus, through measuring the performed state of a $\mathrm{MH}$ tank during charging or discharging process, its SOC can be estimated.

\section{On-line SOC estimation with a state classifier}

\subsection{Framework of the process}

The SOC estimation using the model presented in Eq. 5 is only adapted to a well-controlled experimental condition in the laboratory. The situation in practical application of the MH tank is more complicated, especially in transportation using. Taking the embedded MH hydrogen storage tank on a driving fuel cell vehicle as an example, the usage is under the requirement of driving mission. A whole continually charging or discharging process may not occur, namely, the discharging process might start from an uncertain SOC instead of 
$100 \%$. At the beginning period, the gaseous hydrogen in the tank raised rapidly and the performance is mainly determined by the pressure difference between inside and outside of the tank. Therefore, the fault of SOC estimation cannot be avoided at this stage. When the dehydrogenating reaction is stable, the physical state of the tank is mainly depending on the equilibrium condition presented as P-C-T curves, which means that the pressure and temperature in this stage is determined by the hydrogen concentration. The SOC of the MH tank can be estimated with high accuracy using the proposed model in Eq. 5 . Thus, the current state of reaction should be firstly recognized.

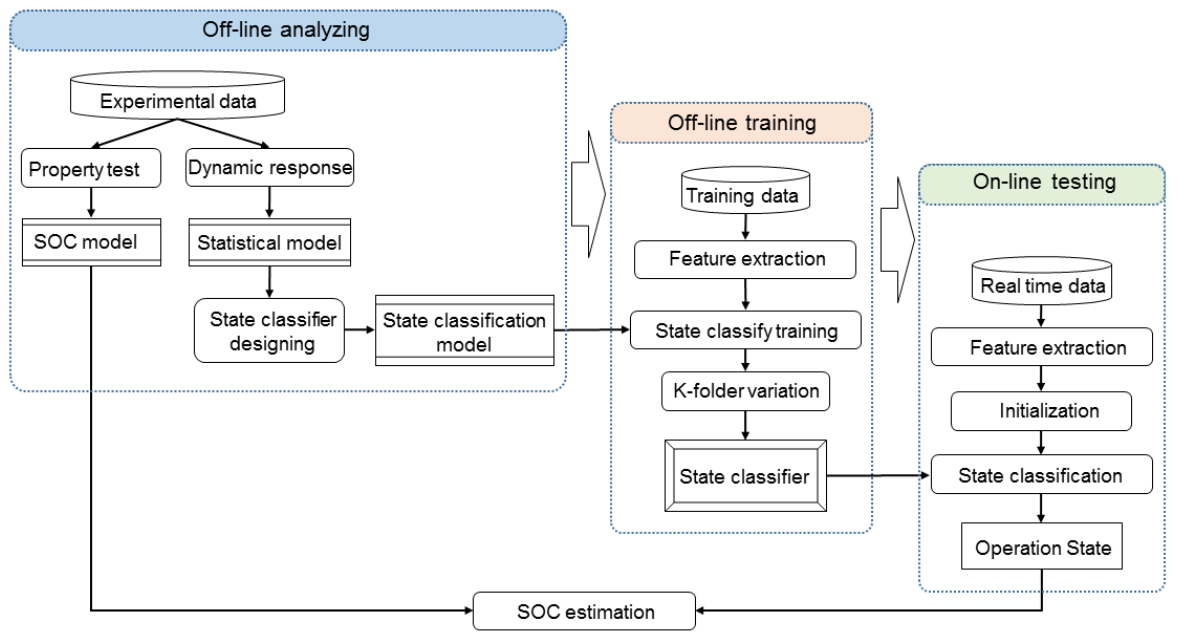

Fig. 1 - Framework of the on-line SOC estimation with a state classifier.

Fig. 1 gives the schematic of the framework for on-line SOC estimation. In this process, based on the property analysis of the reaction, a state classifier is firstly designed to identify the physical state of the $\mathrm{MH}$ reaction bed inside tank during operation. Then, after training the state classifier off-line using the historical database, it could characterize the real data recorded during operation into its relating stage. This process can be realized on-line. Finally, the hydrogen concentration of a $\mathrm{MH}$ tank can be calculated rapidly to estimate the SOC on real time. 
The off-line stage focuses on the historical database analysing and prepossessing. In order to classify the state in a high speed, the optimal feature vectors are required to be extracted from the database. The statistical characters reflecting the properties including slope, kurtosis, entropy, etc, are of great potential as the feature vectors for classify. Afterwards, the state classifier is trained by the training set and adjusted by the testing set of feature vectors. Then, when the well trained classifier comes to the on-line stage, the feature vectors extracted from the real measured database is able to be classified. Therefore, the SOC of the tank can be finally estimated. In this process, classifier is a model set describing the characteristics and features of the database, which can be used to identify the category of the unknown data, namely, map the unknown state to a discrete classification set. The data-based classification method includes fuzzy logic identification, neural network, Bayes classify, support vector machine (SVM) and so on. In this work, a joint multi-classifier is designed through combining the Naive Bayes (NB) classifier and SVM classifier. The detailed information will be presented below.

As discussed above, the hydrogenation reaction is stable in the second stage. In addition, the hydrogen concentration can be achieved by the dynamic performance of pressure and temperature. The stored hydrogen mass can therefore be calculated by the proposed mathematical model, and the SOC of the MH hydrogen storage tank can be estimated.

\subsection{Design of the state classifier}

As mentioned above, a joint multi-classifier constructed by a basic NB classifier and a multi SVM classifier are designed for state classification and identification of the $\mathrm{MH}$ tank reaction process.

NB classifier is developed based on the Bayes statistical theory, which can be used to identify which category the observer belongs to. It has been widely applied in many situations thanks to its high efficiency, high precision, and solid theoretical foundation [26]. In practical applications, the application scope is limited since it's hard to get the prior probability and the class conditional 
probability density of each category. However, with appropriate independence assumption, a smallest misclassification rate can still be achieved using NB classifier. SVM is a robust machine learning model that shows high accuracy with different classification problems [27]. The accuracy of classification is guaranteed for the high dimensional spaces and complex interaction characteristics. The limitation of this method is that only two category classification problems can be solved, which limits the application in complex state classification. Thus, a multi SVM classifier is designed, which can decompose the multi-classification problem into several number of two-classification problems. Through decomposition and reconstruction, two-classification problems can be solved respectively, and the optimal results can then be determined. For the multi-classification problem with the category number of $c$, the SVM classifier is constructed between each category. Therefore, the required SVM classifier number is $c(c-1) / 2$. The training samples of each SVM classifier are two related categories. The voting method is used to determine the classification results that the category got the maximum votes is the class that test sample belongs to. This kind of multiclassifier has significant advantages. Since each SVM classifier only considers two types of samples, the training process is simple to be achieved. Using the majority voting method for making the final decision is easy to implement with a high speed. Meanwhile, the classification accuracy is high. However, when there are many categories to be distinguished, the number of SVM classifiers increases sharply, which affects the training and testing speed, the accuracy might be decreased as well.

Normally, the multi-classification model is integrated by the simple classifiers in two ways that they connected in series or in parallel. In the series multi-classifier, the classification information is transferred from the previous simple classifier to the next one, which means the results of the previous simple classifier and the other input information are combined as the input of the next simple classifier. While in parallel multi-classifier, each simple classifier operates separately and the classification results are concluded in the end. Thus, its speed of classification is significantly increased than that of series multi-classifier. 


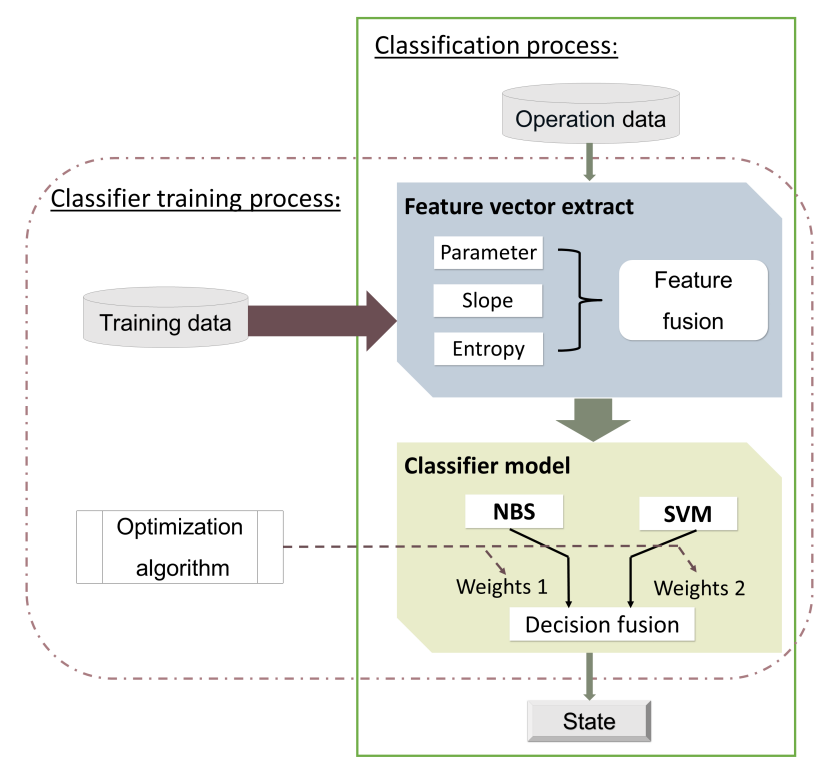

Fig. 2 - Flow chart of joint multi-classification.

What's more, the results of each simple classifier can be complementary. However, if the simple classifiers and the combination rules are not properly selected, the results of the parallel multi-classifier may not rise but fall.

In this study, a joint multi-classifier in parallel structure is designed for the purpose of fully demonstrating the advantages while suppressing the weakness of each single basic classifier. Fig. 2 gives the flow chart of this joint multi-classifier. The multi SVM classifier and NB classifier are trained by the historical data separately, the results of each simple classifiers are afterwards learned and remembered. An optimization algorithm is used to search an optimal weight for the weighted summation of each simple classifier output result on the measurement layer. The final classification result is therefore be determined. In this process, the combination weight is effected by the classification ability of simple classifiers and the state characteristics of the analyzed system. 


\section{Experiments and validation}

4.1. SOC estimation on test bench

\subsubsection{Experiments on the test bench}

In our work, a test bench is built to validate the P-T-C based model for SOC estimation in the laboratory. As presented in Fig. 3, two MH hydrogen storage tanks are connected in parallel as the testing object. The properties and reaction state are monitored and recorded. At the outlet of the tank, a gas flow sensor is installed to record the hydrogen flow rate in and out the tank. Besides, a pressure sensor is put at the tank gate to test the pressure inside the tank. Two temperature sensors are attached at the surface of the MH tanks and the measured results are considered as the reaction bed temperature. During the reaction, the fan matrix, heater and circulation water operates together to control the thermal condition. The fan matrix is used for heat transfer and heat dissipation when the temperature is too high, while the heater is used to warm the circulation water to provide more energy for reaction. Fig. 4 gives the photo of the test bench.

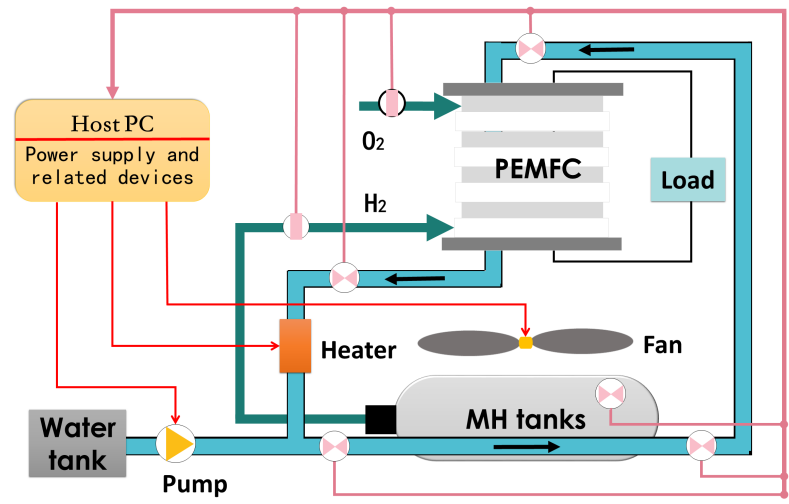

Fig. 3 - Schematic of the test bench. 


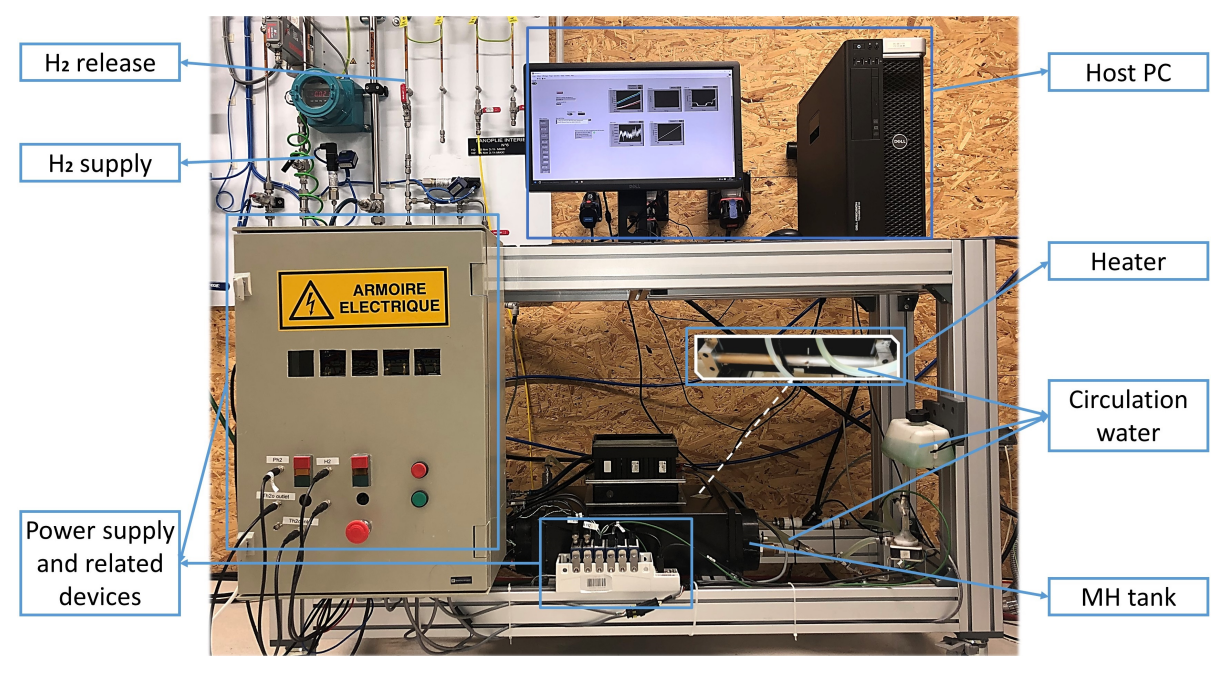

Fig. 4 - Experimental setup.

During the test, the hydrogen is provided by the hydrogen tube in laboratory with the pressure of 7.5bar. The discharged hydrogen from the MH tank is released to the air circulation system. In other words, the hydrogen flow rate input and output of the $\mathrm{MH}$ tank is determined by the differential pressure. The experimental process is controlled by temperature. The temperature inside the test room is always kept as $19^{\circ} \mathrm{C}$.

\subsubsection{SOC estimation in absorption case}

When charging the tanks, the generated heat raises the temperature of $\mathrm{MH}$ tanks. When the measured temperature reaches $26^{\circ} \mathrm{C}$, the charging flow is stopped manually and the fan matrix is turned on to remove the heat. Until the MH tank temperature drop to $19^{\circ} \mathrm{C}$, the charging process is restarted. This process repeats several times until the internal and external pressure is balanced, which means the MH tanks are fully charged. In contrary, the desorption reaction is an endothermic reaction that the $\mathrm{MH}$ tank temperature decreases during discharging. The circulation water is warmed by heater in order to raise the temperature of reaction bed. The temperature threshold is set as $22^{\circ} \mathrm{C}$ to $26^{\circ} \mathrm{C}$. 
From the database obtained on the test bench, the pressure and temperature under certain equilibrium state can be extracted. Then the SOC of the MH tank can be figured out through the statistical model presented in Eq. 5

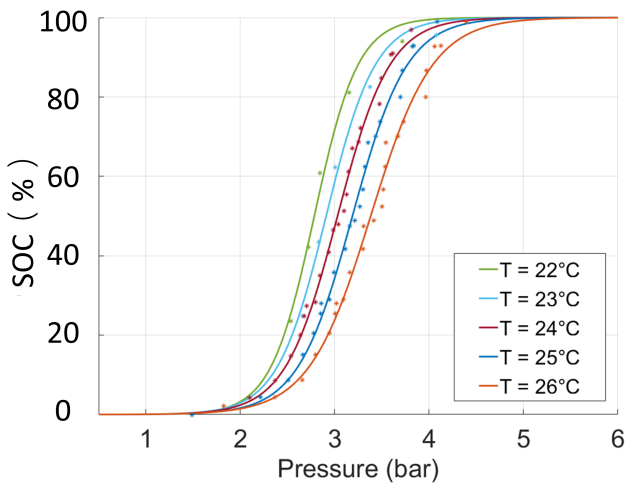

Fig. 5 - Parameter identification of the charging model.

Fig. 5 shows the identification results of the statistical model for SOC estimation of embedded MH tank during the whole process of absorption. As can be seen from each isotherm curve, the absorption process mainly occurred during the pressure varies from $2 b a r$ to 5 bar. When the hydrogen concentration is too low, hydrogen cannot be absorbed. The hydrogenation process will start after the concentration reaches a certain value. After the $\mathrm{MH}$ material in the tank was fully charged, the pressure rises steeply with the increased concentration of gaseous hydrogen. Table 2 presents the coefficients $f_{1}(T)$ and $f_{2}(T)$ used to draw these curves of the model in Fig. 5. The errors between the proposed model and the experimental data are acceptable that the maximum and the minimum value are $9.6 \%$ and $2.5 \%$.

The hydrogen mass put into the MH tank is calculated by the measured hydrogen flow rate. Taking the calculated SOC as the calibration for evaluating the estimation results. From Fig. 5 one can see that the deviation of SOC estimation under different pressure are concentrated in the pressure zone of less than $2 b a r$, where the hydrogen concentration is not high enough and the main 
absorption reaction is not carried out. Moreover, at the end period of charging process, the absorption speed is slow down and the gaseous hydrogen leads to the rapid increase of pressure. Thus, the SOC estimation based on the statistical model is not reliable enough when the pressure is high than 5bar.

Table 2 - Identified parameters for absorption model.

\begin{tabular}{cccc}
\hline \hline$T$ & $f_{1}(T)$ & $f_{2}(T)$ & Error \\
\hline $22^{\circ} \mathrm{C}$ & -4.33 & 12.05 & $5.07 \%$ \\
$23^{\circ} \mathrm{C}$ & -3.85 & 11.19 & $5.81 \%$ \\
$24^{\circ} \mathrm{C}$ & -3.61 & 10.92 & $8.74 \%$ \\
$25^{\circ} \mathrm{C}$ & -3.44 & 10.98 & $8.33 \%$ \\
$26^{\circ} \mathrm{C}$ & -3.05 & 11.28 & $9.12 \%$ \\
\hline \hline
\end{tabular}

In the SOC model, $f_{1}(T)$ and $f_{2}(T)$ reflect the influence of temperature on the equilibrium pressure, which will determine the shape of P-C-T curves of the reaction. The values of equilibrium pressure under each temperature could also be obtained by fitting the functions of $f_{1}(T)$ and $f_{2}(T)$. Through identifying how the coefficients variation with different temperatures, the function of $f_{1}(T)$ and $f_{2}(T)$ are identified as follows:

$$
\begin{gathered}
f_{1}(T)=0.29 * T-10.6 \\
f_{2}(T)=-0.368 * T+19.1
\end{gathered}
$$

\subsubsection{SOC estimation in desorption case}

Similar to the absorption case, the data points of pressure under certain temperature during hydrogen desorption process can be extracted. Fig. 6 presents the results of the mathematical models representing the hydrogen concentration stored in a $\mathrm{MH}$ hydrogen storage tank during desorption process, all these models are identified under different temperature while in one cycle. 


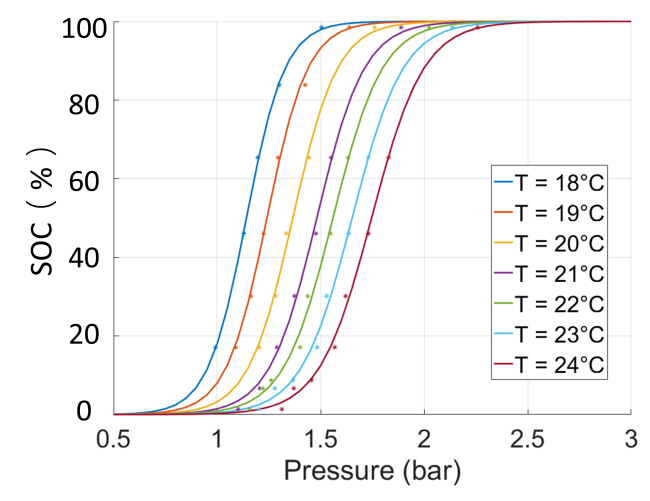

Fig. 6 - Parameter identification of the discharging model.

Obviously, the desorption process is normally carried out in a lower pressure zone than that of absorption. For the tested tank, the pressure is between 1 bar to 2bar. At the end period of discharging process, because of the small hydrogen concentration and low pressure, the desorption reaction cannot keep going automatically. Special measures are necessary to fully discharge a $\mathrm{MH}$ hydrogen storage tank like heating. However, during practical application the high temperature can not be achieved. The hydrogen retention in the tank will lead to the capacity degradation of an embedded MH tank after long time usage, which deserves special attention, which will be discussed later. The coefficients of the model for desorption process are presented in Table 3, in which the maximum error is as low as $2.47 \%$. Similar to the charging process, the errors focus on the low pressure situation, namely, higher difficulty is inevitable for SOC estimation at low hydrogen concentration. 
Table 3 - Identified parameters of SOC estimation model for desorption process.

\begin{tabular}{cccc}
\hline \hline$T$ & $f_{1}(T)$ & $f_{2}(T)$ & Error \\
\hline $18^{\circ} \mathrm{C}$ & -10.74 & 12.26 & $1.14 \%$ \\
$19^{\circ} \mathrm{C}$ & -10.14 & 12.58 & $2.73 \%$ \\
$20^{\circ} \mathrm{C}$ & -9.29 & 12.69 & $4.55 \%$ \\
$21^{\circ} \mathrm{C}$ & -8.72 & 12.92 & $9.56 \%$ \\
$22^{\circ} \mathrm{C}$ & -8.49 & 13.23 & $9.36 \%$ \\
$23^{\circ} \mathrm{C}$ & -8.22 & 13.58 & $9.35 \%$ \\
$24^{\circ} \mathrm{C}$ & -800 & 13.97 & $9.21 \%$ \\
\hline \hline
\end{tabular}

The function of $f_{1}(T)$ and $f_{2}(T)$ in $S O C$ model during discharging process are identified as follows:

$$
\begin{aligned}
& f_{1}(T)=0.4782 * T-19.1 \\
& f_{2}(T)=0.2422 * T+7.9
\end{aligned}
$$

\subsection{On-line SOC estimation in real operation case}

\subsubsection{Validation procedure}

The online SOC estimation method, that combines the state classifier and SOC model, is verified on the database of the real fuel cell hybrid electrical vehicle (FCHEV) test [28. In this project, ten FCHEVs are designed and operated. On these vehicles, two MH hydrogen storage tanks, same as the ones tested in laboratory, are connected in parallel to store hydrogen and supply the embedded fuel cell. The refuelling station provides gaseous hydrogen flow with constant pressure 10bar to charge the MH tank. During operation, the fuel cell system on the FCHEVs is used as the first power source to charge the batteries and then supply the vehicle load. Therefore, stable hydrogen flow is required 
(a)

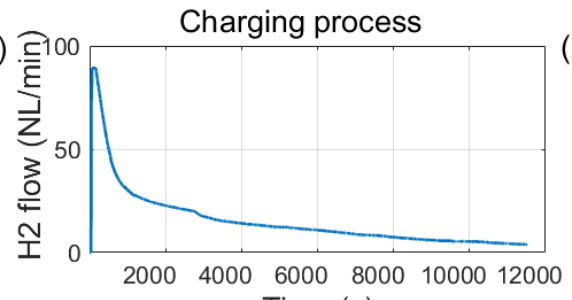

(c)

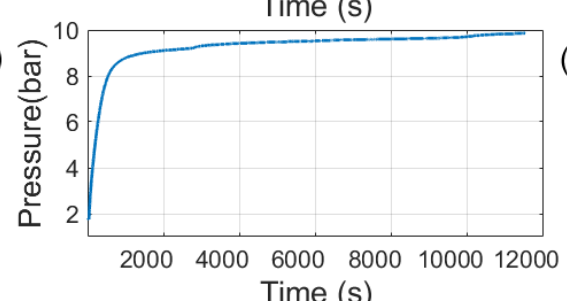

(e)

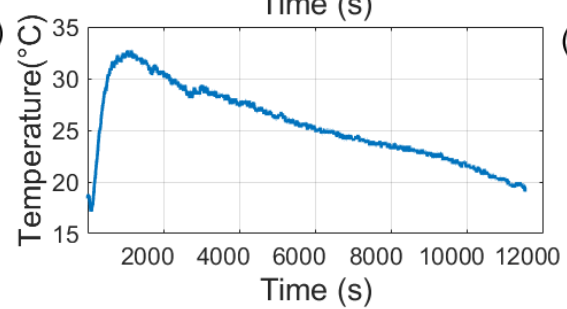

(b) $\widehat{40}$ Discharging process

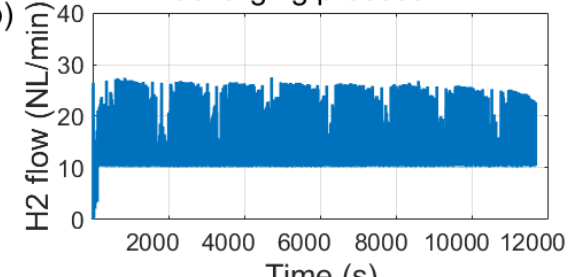

(d)

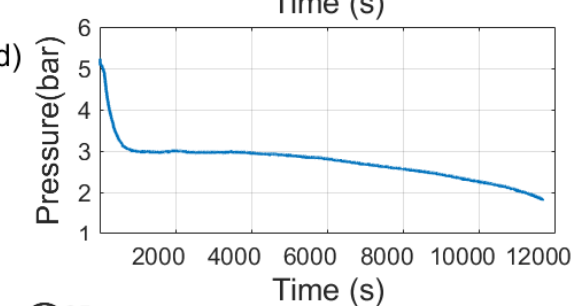

(f)

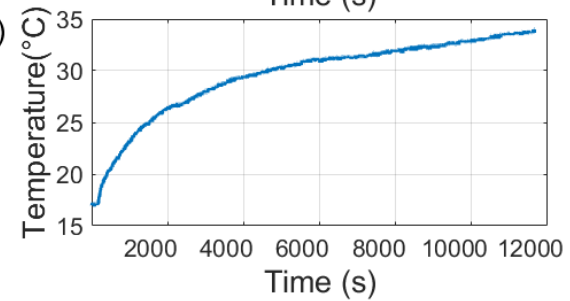

Fig. 7 - Physical state variation: (a)Hydrogen flow variation during charging process; (b)Hydrogen flow variation during discharging process; (c)Pressure variation during charging process; (b)Pressure variation during discharging process; (e)Temperature variation during charging process; (f)Temperature variation during discharging process.

Fig. 7 show the examples of the dynamic response of the MH hydrogen storage tanks. During charging process, the physical state variation presents two different stages. The first stage is dominated by the pressure difference so that the pressure and temperature increased rapidly with the high gaseous hydrogen flow imported to the tank. Then the hydrogen is absorbed by MH host material continuously, which leads to the pressure and temperature tend to constant. 
In this stage, the temperature decreases due to the heat remove of the thermal control system, which is used for accelerating the hydrogenation reaction. Compared to the three hydrogenation reaction phases introduced above, these two stages of practical application are also driven by external factors like pressure. No matter of the initial hydrogen remaining in the tank, the performance of the first stage shows the same tend. When the gaseous hydrogen concentration meets the hydrogenation conditions, the second stage starts, which will be determined by the hydrogen to metal ratio.

The discharging process is separated into three stages. The hydrogen release flow rate is controlled to be constant to meet the requirement of fuel cell consumption. The thermal control system provide stable energy to heat the tank. Similar to charging process, due to the pressure deference, the hydrogen pressure drops quickly in the first stage. The temperature of MH tank raised rapidly because of the low hydrogen generation reaction speed. When the hydrogen desorption reaction occurred stable and rapidly, the hydrogen generation speed is high enough to satisfy the releasing requirement so that more heat is absorbed for reaction. Thus, in the second stage, the pressure is maintained stable and the temperature raised speed is slow down. The performance of third stage is determined by the low SOC of MH tank. During the sorption procedure, the equilibrium pressure drops at low hydrogen concentration, which also leads to the decreased hydrogen generation speed. In order to hold the hydrogen releasing speed, more heat absorption is needed. As a result, the pressure drops and the rate of temperature raise decreases at the third stage.

The feature vectors selected for classification can reflect the characteristics of each physical state in different stage. The time domain features of these data based on statistical characteristics are typical for the performance since the recorded data is time sampled. The energy density variation could also present the characteristic of the data. Therefore, in this work, kurtosis feature and entropy feature are also picked as classification feature vectors.

The optimal weight is searched during classifier training process using Grid Search (GS) method. GS is an exhaustive search method that tests all the 
set of the candidates' weights to find out the best performing one as the final result. Thus, the optimal weight with highest classification precision is then obtained. Since the number of categories is not too much, the disadvantage of time consuming can be ignored.

Actually, both two single classifiers have a good consequent on the state classification of hydrogenation and dehydrogenating process, and the classification accuracy rate of the tested databases have reached more than $70 \%$. The NB classifier has a simple structure, so the time required for classifier training and state classification of each test data group is short. While the multiple SVM classifier is composed of more than one single SVM classifiers, in which each state category of the data is trained in pairs and takes a relatively long time. However, the correct recognition rate of the test database by the joint multiclassifiers is much higher than that of each single classifier, which has higher application value. In the joint multi-classifier training, the GS algorithm needs to perform multiple iterations when seeking the optimal weight, which leads to a significant increase in training time compared to the single classifier. However, the classification time for each set of test data is still short, which can meet the time limit in practical applications.

\subsubsection{Method evaluation}

In order to evaluate the proposed on-line SOC estimation method, ten group of real operation data are picked form charging and discharging database randomly for validation. K-folds cross validation method is applied for testing the classification accuracy of the designed joint multi-classifier. In each test procedure, 9 groups of data are set as the training set and the other one is set for testing. After repeating the procedure for 10 times, the average value of the mean square error of all the test procedure is regarded as the error of the classifier.

For the SOC estimation of charging process, the combine weight of NB classifier and SVM classifier are 0.5. Under this situation, the highest state classification accuracy of $91.3 \%$ is achieved. While for discharging process, the 
highest accuracy of state classification using joint multi-classifier is $83.2 \%$ with the combine weight of 0.6 and 0.4 , respectively for NB classifier and SVM classifier.

\subsubsection{On-line SOC estimation during vehicle charging}

As discussed above, the on-line SOC estimation during charging and discharging process are quite difficult but important. Several groups of real operation data from Mobypost database are selected to validate the proposed on-line SOC estimation algorithm. In the used database for charging process, the data was recorded in refuelling station. On the vehicle management system, the hydrogen mass filling into the tank is calculated by the information from hydrogen flow rate, which is used for calibrating the on-line estimation result.

Fig. 8(a) presents the state classification result during one charging process. Obviously, the classification error is merely appears in transition of the first stage to the second stage, which provides the possibility to estimate the SOC using the statistical model in accuracy.

Fig. 8(b) gives the on-line SOC estimation result. Here, the source of the picked data for validation is a charging process from completely released state to fully charged state occurred on one FCHEV of Mobypost project. In this project, the fuel cell mode stops when the SOC of the MH tank is lower than $10 \%$ for protecting fuel cell. As a result, the initial SOC is set as $10 \%$. As discussed above, the SOC estimation based on the proposed statistical model is inaccuracy. Therefore, when the output of the on-line state classifier shows the reaction is under the first stage, the SOC is calculated by the integration with time of the hydrogen flow rate entered. Taking the calculated value on the vehicle as calibration, the SOC estimation error is $0.2 \%$. The fluctuation of ambient temperature and the measurement deviation might be the main reason causes the estimation error. 

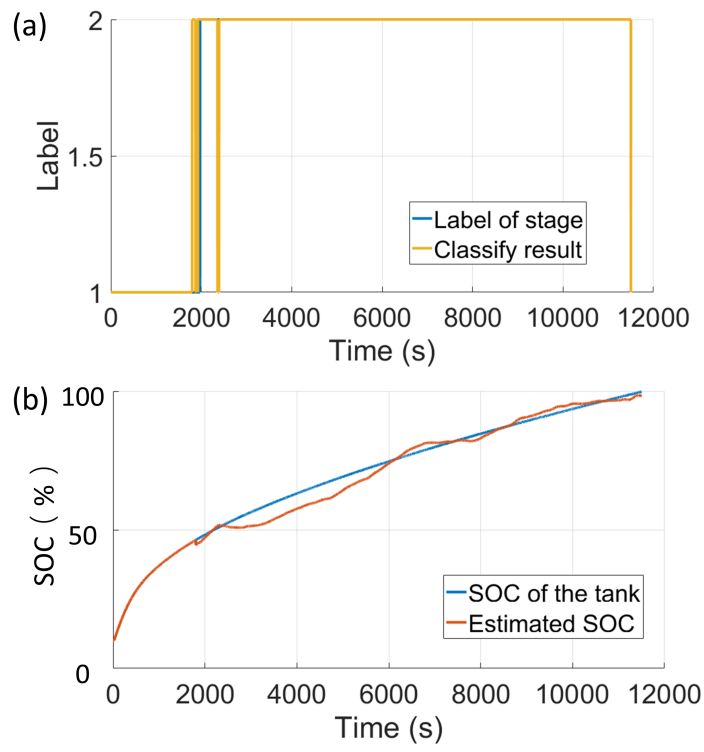

Fig. 8 - On-line test results for charging process. (a)On-line state classification result; (b)SOC estimation result.

\subsubsection{On-line SOC estimation during vehicle operation}

The discharging process is more complicated than charging, while it is more significant to estimate the SOC of embedded MH tank during vehicle operation.

The used database for validation was recorded in a continuously vehicle operation process, before that the hydrogen storage tank is fully charged at refuelling station. Therefore, the recorded data of remaining hydrogen mass is calculated by the released hydrogen flow rate.

Fig. 9(a) presents the on-line state classification result of the joint multiclassifier. It can be seen that the classification error is concentrated in the first stage and the third stage, while in the second stage, the performance of the classifier is excellent. 

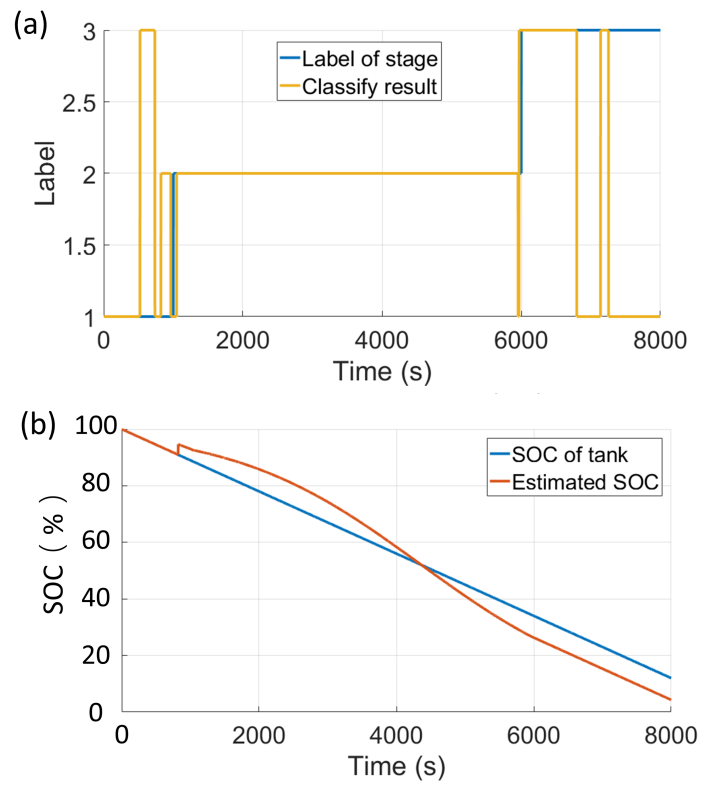

Fig. 9 - On-line test results for discharging process. (a)On-line state classification result; (b)SOC estimation result.

The classification result is used to estimate the SOC of the embedded MH hydrogen storage tank. Based on the mathematical model proposed above, the SOC of the tank can be calculated by the data point recognized as second stage. Since the degradation of the $\mathrm{MH}$ hydrogen storage tank is not taken into consideration, the hydrogen mass remained in the tank at the period of recognized second stage can be estimated. Fig. 9(b) gives the estimation result, in which the hydrogen mass in the first stage and third stage is calculated by the recorded data of hydrogen flow rate. Compared to the value of hydrogen mass in the tested database, the on-line SOC estimation results shows a great agreement with the mean square error of $4.29 \%$, which is acceptable. Besides, the real operation data is recorded every second, and the testing time for one group of data is less than $1 \times 10^{-4} s$. Thus, this on-line SOC estimation with high accuracy and low time consumption is of high practical value. 


\section{Conclusion}

This work focuses on the data based study of the MH hydrogen storage tank. The studied database is collected from laboratory experiments and Mobypost project separately, which are representative for reflecting the performance of the hydrogenation reaction. The models designed in this work is based on the statistical theory to characterize the performance of the main parameters of reaction including pressure, temperature, hydrogen flow rate and hydrogen mass. The P-C-T based SOC estimation method is proposed on the basis of the main physical character of the reaction process. More data in a wide range of distinct temperatures and long-term tests will be of great help to validate or improve the proposed model. In addition, an effective on-line SOC estimation method is proposed through designing a joint multi-classifier to recognize the stage of reaction. Combined with the P-C-T based SOC model, the hydrogen remaining in the tank can be estimated on real time. This method might be not an accurate measurement of hydrogen sorption. However, it gives a solution for on-board hydrogen storage SOC estimation, which can provide significant information for the embedded energy management system.

\section{Acknowledgement}

The support from Chinese Scholarship Council (CSC), MobyPost project funded under the Grant Agreement No.256834 by the European Union's seventh Framework program (FP7/2007e2013) for the Fuel Cell and Hydrogen Joint Technology Undertaking (http://mobypostproject.eu/) and OenVHy project funded by the Bourgogne-Franche comte region-France are gratefully acknowledged.

\section{References}

[1] G. Dolf, B. Francisco, S. Deger, B. Morgan, D, W. Nicholas, G. Ricardo, The role of renewable energy in the global energy transformation, Energy Strategy Reviews 24 (2019) 38-50. 
[2] S. Sharma, S. K. Ghoshal, Hydrogen the future transportation fuel: From production to applications, Renewable and Sustainable Energy Reviews 43 (2015) 1151-1158.

[3] S. Mcdonagh, S. Ahmed, C. Desmond, J. D. Murphy, J. Yan, Hydrogen from offshore wind: Investor perspective on the profitability of a hybrid system including for curtailment, Applied Energy 265 (2020) 114732.

[4] Yang, Jun, Sudik, Andrea, Wolverton, Christopher, Siegel, J. Donald, High capacity hydrogen storage materials: attributes for automotive applications and techniques for materials discovery, Chemical Society Reviews 39 (2010) 656-675.

[5] P. R. Prabhukhot, M. M. Wagh, A. C. Gangal, A review on solid state hydrogen storage material.

[6] M. Marinelli, M. Santarelli, Hydrogen storage alloys for stationary applications, The Journal of Energy Storage 32 (10) (2020) 101864.

[7] N. R. J. Hynes, R. Sankaranarayanan, P. S. Kannan, A. Khan, H. Dzudzevic-Cancar, Solid-state hydrides for hydrogen storage, 2021.

[8] S. Koohi-Fayegh, M. A. Rosen, A review of energy storage types, applications and recent developments, The Journal of Energy Storage 27 (2020) 101047.

[9] T. Wei, K. L. Lim, Y. Tseng, S. Chan, A review on the characterization of hydrogen in hydrogen storage materials, Renewable and Sustainable Energy Reviews 79 (2017) 1122-1133.

[10] T. Blach, E. M. Gray, Sieverts apparatus and methodology for accurate determination of hydrogen uptake by light-atom hosts, Journal of Alloys and Compounds 446 (2007) 692-697.

[11] C. Webb, E. M. Gray, The effect of inaccurate volume calibrations on hydrogen uptake measured by the sieverts method, International Journal of Hydrogen Energy 39 (5) (2014) 2168-2174. 
[12] C. Webb, E. M. Gray, Analysis of the uncertainties in gas uptake measurements using the sieverts method, International Journal of Hydrogen Energy 39 (1) (2014) 366-375.

[13] F. A. Stevie, C. Zhou, M. Hopstaken, M. Saccomanno, Z. Zhang, A. Turansky, Sims measurement of hydrogen and deuterium detection limits in silicon: Comparison of different sims instrumentation, Journal of Vacuum Science \& Technology B, Nanotechnology and Microelectronics: Materials, Processing, Measurement, and Phenomena 34 (3) (2016) 03H103-1-3H1035.

[14] C. Wan, R. Denys, V. Yartys, In situ neutron powder diffraction study of phase-structural transformations in the lamgni battery anode alloy, Journal of Alloys and Compounds 670 (2016) 210-216.

[15] I. Agency, Role of nuclear based techniques in development and characterization of materials for hydrogen storage and fuel cells, Role of nuclear based techniques in development and characterization of materials for hydrogen storage and fuel cells, 2012.

[16] L. Xi, Z. Qi, L. Haiyan, Y. Hongguang, L. Tao, L. Qian, Numerical analysis of the effects of particle radius and porosity on hydrogen absorption performances in metal hydride tank - sciencedirect, Applied Energy 250 (2019) 1065-1072.

[17] S. Mohammadshahi, E. M. Gray, C. Webb, A review of mathematical modelling of metal-hydride systems for hydrogen storage applications, International Journal of Hydrogen Energy 41 (5) (2016) 3470-3484.

[18] K. Minko, V. Artemov, G. Yan'kov, Numerical simulation of sorption/desorption processes in metal-hydride systems for hydrogen storage and purification. part ii: verification of the mathematical model, International Journal of Heat and Mass Transfer 68 (2014) 693-702. 
[19] A. Jemni, S. B. Nasrallah, Study of two-dimensional heat and mass transfer during absorption in a metal-hydrogen reactor, International Journal of Hydrogen Energy 20 (1) (1995) 43-52.

[20] A. Freni, F. Cipiti, G. Cacciola, Finite element-based simulation of a metal hydride-based hydrogen storage tank, International Journal of Hydrogen Energy 34 (20) (2009) 8574-8582.

[21] J. Nam, J. Ko, H. Ju, Three-dimensional modeling and simulation of hydrogen absorption in metal hydride hydrogen storage vessels, Applied energy 89 (1) (2012) 164-175.

[22] D. Arora, C. Bonnet, M. Mukherjee, S. Rael, F. Lapicque, Direct hybridization of pemfc and supercapacitors: Effect of excess hydrogen on a single cell fuel cell durability and its feasibility on fuel cell stack, Electrochimica Acta 310 (2019) 213-220.

[23] A. Ebadighajari, J. DeVaal, F. Golnaraghi, Optimal control of fuel overpressure in a polymer electrolyte membrane fuel cell with hydrogen transfer leak during load change, Journal of Power Sources 340 (2017) 247-257.

[24] D. Chabane, F. Harel, A. Djerdir, D. Candusso, O. Elkedim, N. Fenineche, A new method for the characterization of hydrides hydrogen tanks dedicated to automotive applications, international journal of hydrogen energy 41 (27) (2016) 11682-11691.

[25] D. Zhu, Y. Ait-Amirat, A. N'Diaye, A. Djerdir, New dynamic modeling of a real embedded metal hydride hydrogen storage system, International Journal of Hydrogen Energy 44 (55) (2019) 29203-29211.

[26] Y.-C. Zhang, L. Sakhanenko, The naive bayes classifier for functional data, Statistics \& Probability Letters 152 (2019) 137-146.

[27] V. Vapnik, The nature of statistical learning theory, Springer science \& business media, 2013.

[28] http://mobypost-project.eu. 